\title{
Graphene as Dry Adhesive Interacting with Semiconductor Substrates
}

\author{
Jun Sun, Tao Xu, Feng Xu, Xiao Xie, Litao Sun ${ }^{*}$ \\ SEU-FEI Nano-Pico Center, Key Laboratory of MEMS of Ministry of Education, Southeast University, Nanjing, China \\ *Corresponding Author: Litao Sun, slt@,seu.edu.cn
}

\begin{abstract}
Here we show by molecular dynamics that graphene could be utilized as dry adhesive interacting with semiconductor substrates. Various potential semiconductor substrates are introduced and present similar results. This work opens up a new prospect for the applications of graphene and would be valuable for the scientific studies of adhesion at nanoscale.
\end{abstract}

Keywords-graphene; adhesion; semiconductor substrates; molecular dynamics

\section{INTRODUCTION}

As a single layer of atoms with strong covalent bond, graphene is attractive for nanoelectromechanical systems (NEMS) due to its extraordinary high Young's modulus $(\sim 1000 \mathrm{GPa})$, tensile strength $(\sim 100 \mathrm{GPa})$, high electron mobility, tunable band gaps and high thermal conductivity [1]. On the other hand, its extreme thin thickness inevitably induces flexibility and in-plane rippling even at room temperature $[2,3]$. This feature is beneficial for its interaction with substrate because of more contact areas.

Recently, the ultrastrong adhesion of graphene with $\mathrm{SiO}_{2}$ substrate is reported [4]. It is found that the measured adhesion energies of monolayer graphene and multilayer graphene are as high as $0.45 \mathrm{~J} \mathrm{~m}^{-2}$ and $0.31 \mathrm{~J} \mathrm{~m}^{-2}$, which are larger than the adhesion energy measured in typical micromechanical structures and are comparable to solid-liquid adhesion energies. Such ultrastrong adhension can be attributed to the extreme flexibility of graphene, which allows it to conform to the topography of even the smoothest substrates, thus making its interaction with the substrate more liquid-like than solidlike. The ultrastrong adhesion inspires us to explore graphene as dry adhesive for potential application in NEMS, such as dry adhesives and wafer bonding, just like gecko's foot hair [5].

In this research study, we perform theoretical investigations on graphene interaction with various semiconductor substrates. The results show that graphene is suitable for serving as dry adhesives that are mechanical robust and electrical conductive.

\section{METHODOLOGY}

The atomic interaction were modeled by the COMPASS force field (condensed-phased optimized molecular potential for atomistic simulation studies), which was the first ab initio force field that was parametrized and validated using condensed phase properties. This force field has been proven to be applicable in describing the mechanical properties of carbon nanostructure [6-8]. The force field is expressed as a sum of valence (or bond), cross-terms, and nonbond interactions: $E_{\text {total }}=E_{\text {valence }}+E_{\text {crossterm }}+E_{\text {nonbond. }}$. The energy of valence, $\mathrm{E}_{\text {valence, }}$ is generally accounted for by terms including bond stretching, valence angle bending, dihedral angle torsion, and inversion. The crossterms, $\mathrm{E}_{\text {crossterm, account for factors }}$ such as bond or angle distortions caused by nearby atoms to accurately reproduce the dynamic properties of molecules. The energy of interactions, $\mathrm{E}_{\text {nonbond }}$, between nonbonded atoms is primarily accounted for by van der Waals effect and hydrogen bond effect.

As shown in Figure 1, atomic models of graphene nanoribbon ( $8 \mathrm{~nm}$ in length, $2 \mathrm{~nm}$ in width) were created and combined with atomic models of silicon (100) lattice plane of about $11 \AA \AA$ thickness.

The simulations presented herein were modeled by a quasistatic, displacement update scheme. During the simulations, the fixed carbon atoms were moved towards the surface in increments of $0.1 \AA$, then the entire system was allowed to equilibrate for 500 molecular dynamics (MD) steps and moved towards the surface.

The dynamics process is conducted to allow the system to exchange heat with environment at a constant temperature. For a temperature control, the thermodynamic temperature is kept constant by allowing the simulated system to exchange energy with a "heat bath". The Berendsen method is employed to keep the system at the room temperature and a 1 femtosecond time step was used in all MD simulations.

\section{RESULTS AND DisCUSSION}

Snapshots of the graphene nanoribbon deformation above the silicon (100) plane were shown in Fig. 1. When the nanoribbon was moved towards the solid surface, due to the strong van der Waals interaction, the leading edge of the nanoribbon were first attracted by the silicon layer up to a critical position. Then repulsive force pushed them back along the vertical axis. Considering that graphene is an extremely thin material, it can buckle and ripple under thermal undulation even at room temperature. When the graphene was moved further, the repulsive forces were increased and they cause the graphene nanoribbon buckling, bending and slipping. 


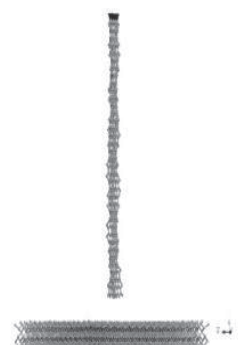

(a) $\triangle Z=0 \AA$

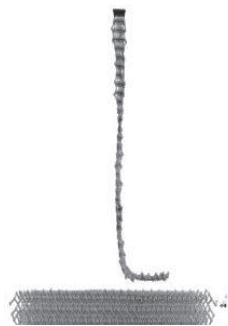

(c) $\triangle Z=20 \AA$

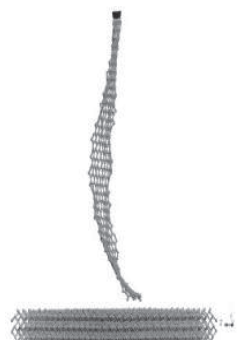

(b) $\triangle Z=10 \AA$

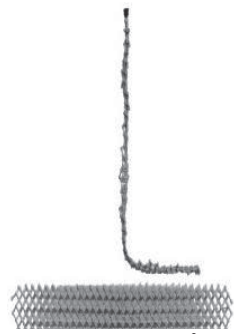

(d) $\triangle Z=30 \AA$
Fig. 1. Simulation snapshots of the adhesion between graphene nanoribbon and Si (100) substrates with (a) $\triangle \mathrm{Z}=0 \AA$, (b) $\triangle \mathrm{Z}=10 \AA$, (c) $\triangle \mathrm{Z}=20 \AA$, and (d) $\triangle Z=30 \AA$.

As it can be seen in Fig. 1(b), the front part of graphene after onset of buckling makes an angle with the silicon surface, exhibiting an "L" shape. After buckling and bending, the slipping of graphene was observed along the horizontal axis. This indicated that the relative motion between graphene and silicon substrates only generated a low friction.

The deformation behavior of graphene nanoribbon in above molecular dynamics simulations revealed a liquid-like behavior other than solid-like behavior [4]. The short distance (about $3.4 \AA$ ) between the nanoribbon and solid surface indicated the adhesion energy was strong. To estimate the adhesion in the interface, the variation of perpendicular component of the total force as a function of displacement in the Z-direction was calculated to be $10^{-1} \mathrm{nN} / \mathrm{nm}^{2}$, which was several hundred times higher than that of gecko's foot hair [5]. It implied that the connection between graphene and silicon substrate was mechanical robust. Since graphene has tunable band gaps, this solid bonding may provide a new direction to three dimensional integrated circuits involving old semiconductor materials (silicon) and next generation semiconductor materials (graphene). Furthermore, the robust bonding may also be utilized into wafer bonding for future nanoelectromechanical systems (NEMS).

The total potential energy and van der Waals interaction energy as a function of $\mathrm{Z}$ displacement were calculated and presented in Fig. 2 and Fig. 3. It was clear that the curve was linear and the drop of total potential energy was equal to the drop of van der Waals interaction energy (here there wasn't hydrogen bond energy component). It was clear that the potential energy dissipation was proportional to the overlapped contact areas, i.e. there was an approximately constant attraction force in the compress process. This meant that the adhesion force between graphene and silicon substrate was constant, thus the adhesion of graphene could be utilized as constant force spring, resembling the carbon nanotube bundles [9-12].
In Fig. 4, the bond length distribution of the graphene nanoribbon in the initial/final configurations were presented. The statistics distribution of bond length mainly concentrated in the vicinity of natural C-C bond length, $1.41 \AA$. However, the distribution range of bond length broadened, which indicated that more $\mathrm{C}-\mathrm{C}$ bonds endured stretching or squeezing strain due to the buckling effect of graphene. The longest C-C bond was still below $1.77 \AA$, which was thought be the critical breaking length for $\mathrm{C}-\mathrm{C} \mathrm{sp}^{2}$ bond in most classic force field [13]. It implied the compress process was mild and lossless.

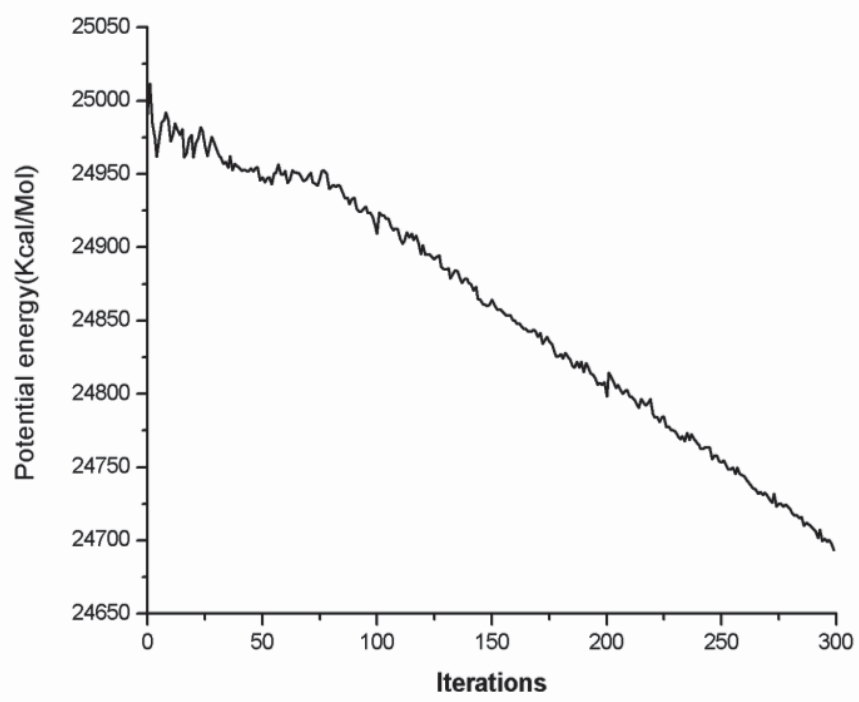

Fig. 2. The system potential energy versus iterations. The displacement of graphene increase in increments of $0.1 \AA$ for each iteration and the total linear decline of energy is about $300 \mathrm{Kcal} / \mathrm{mol}$.

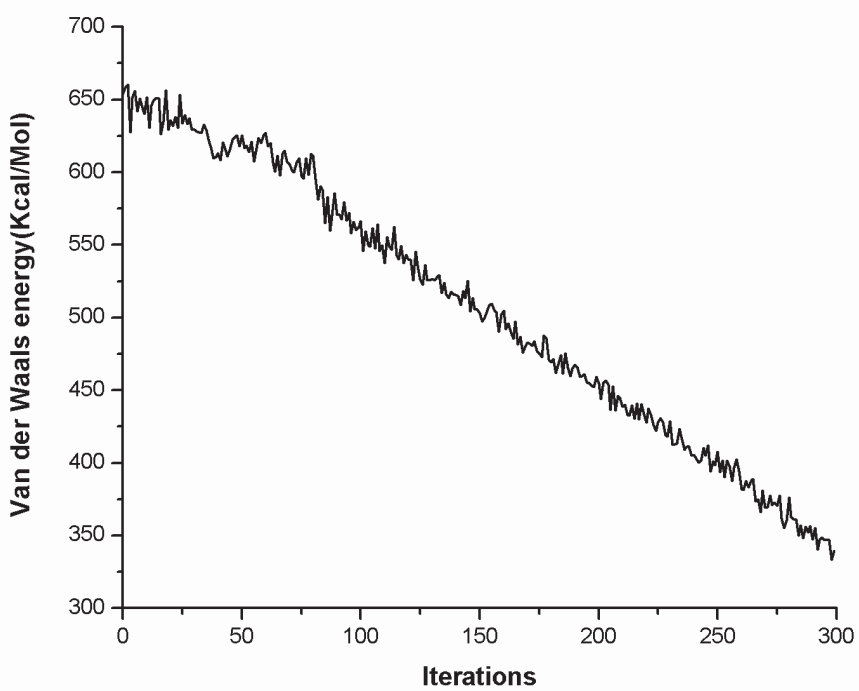

Fig. 3. The van der Waals energy versus iterations. The decline of van der Waals energy is $300 \mathrm{Kcal} / \mathrm{mol}$. 


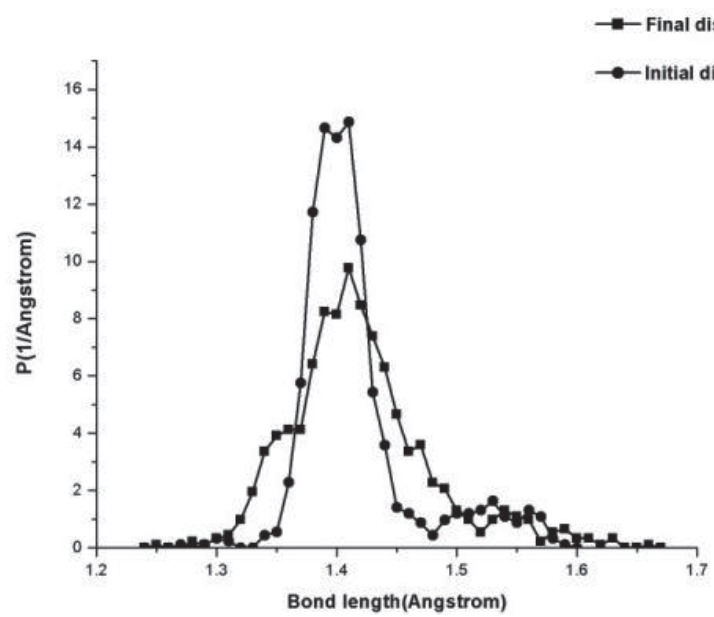

Fig. 4. The bond length distribution of the graphene nanoribbon in the initial/final configurations.

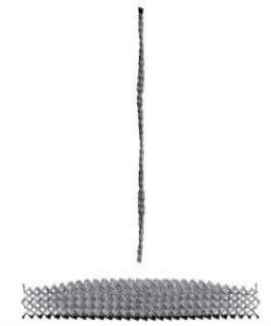

(a) $\triangle \mathrm{Z}=0 \AA$

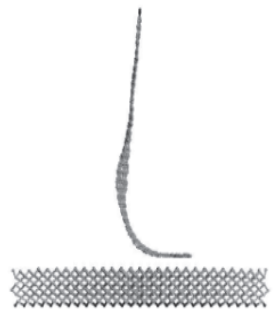

(c) $\triangle \mathrm{Z}=20 \AA$

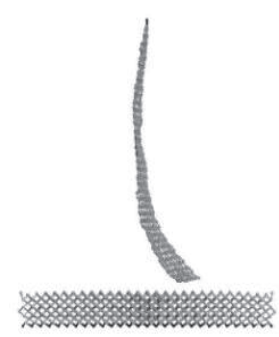

(b) $\triangle \mathrm{Z}=10 \AA$

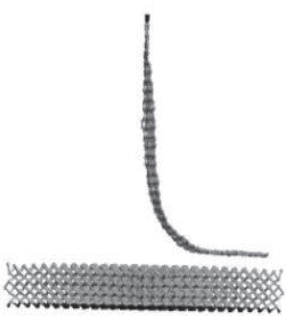

(d) $\triangle \mathrm{Z}=30 \AA$
Fig. 5. Simulation snapshots of the adhesion between graphene nanoribbon and GaAs (100) substrates with (a) $\triangle Z=0 \AA$, (b) $\triangle Z=10 \AA$, (c) $\triangle Z=20 \AA$, and (d) $\triangle Z=30 \AA$.

Finally, several other potential semiconductor substrates (GaAs and $\mathrm{SiC}$ ) were introduced. We set all surfaces with similar size and thickness to avoid their influence and the roughness effect. As shown in Fig. 5 and Fig. 6, the strong adhesion behavior of graphene was universal in the nature.

\section{CONCLUSIONS}

In conclusion, theoretical investigations on the adhesion between graphene and various semiconductor substrates were performed. Atomistic structure changes of graphene nanoribbon were revealed and corresponding potential energy variations were given. The bond length distribution of the graphene nanoribbon in the initial/final configurations indicated that the liquid-like adhesion of graphene is mild and lossless. At last, GaAs and $\mathrm{SiC}$ substrates were introduced. It was found that the strong adhesion behavior of graphene was

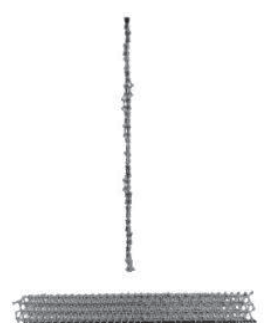

(a) $\triangle Z=0 \AA$

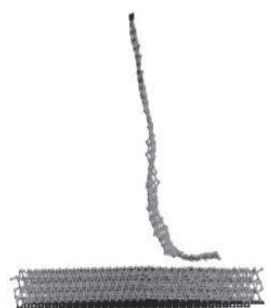

(c) $\triangle Z=20 \AA$

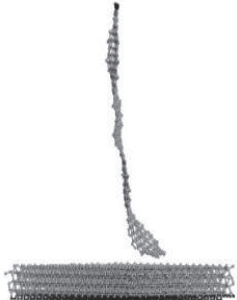

(b) $\triangle \mathrm{Z}=10 \AA$

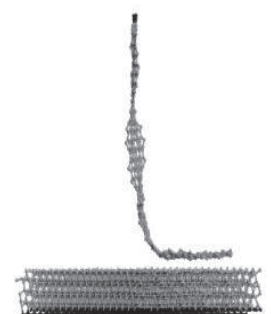

(d) $\triangle Z=30 \AA$
Fig. 6. Simulation snapshots of the adhesion between graphene nanoribbon and $\mathrm{SiC}(100)$ substrates with (a) $\triangle \mathrm{Z}=0 \AA$, (b) $\triangle \mathrm{Z}=10 \AA$, (c) $\triangle \mathrm{Z}=20 \AA$, (d) $\triangle \mathrm{Z}=30 \AA$.

universal in the nature. Our work opens up a new prospect for the applications of graphene and would be valuable for the scientific studies of adhesion at nanoscale.

\section{ACKNOWLEDGMENT}

This work was supported by the National Basic Research Program of China (Grant Nos. 2009CB623702 and 2011CB707601), and the National Natural Science Foundation of China (Nos. 61274114 and 51071044).

\section{REFERENCES}

[1] K.S. Novoselov, A.K. Geim, S.V. Morozov, D. Jiang, Y. Zhang, S.V. Dubonos, I.V. Grigorieva, A.A. Firsov, "Electric Field Effect in Atomically Thin Carbon Films”, Science, Vol. 306, pp. 666-669, 2004.

[2] A. Fasolino, J.H. Los, M.I. Katsnelson, "Intrinsic ripples in graphene", Nat. Mater., Vol. 6, pp. 858-861, 2007.

[3] Z.P. Xu, M.J. Buehler, "Geometry controls conformation of graphene sheets: membranes, ribbons and scrolls", ACS Nano, Vol. 4, pp. 3869 3876, 2010.

[4] S. Koenig, N. Boddeti, M. Dunn, J. Scott, "Ultrastrong Adhesion of Graphene Membranes", Nat. Nanotech, Vol. 6, pp. 543-546, 2011.

[5] K. Autumn, Y. Liang, S. Hsieh, W. Zesch, W. Chan, T. Kenny, R. Fearing, R. Full, "Adhesive Force of A Single Gecko-foot Hair", Nature, Vol. 405, pp. 681-685, 2000.

[6] Q. Wang, "Atomic transportation via carbon nanotubes", Nano Lett., Vol. 9, pp. 245-249, 2009.

[7] K.Y. Yang, Q.Z. Xue, D. Xia, H.J. Chen, J. Xie, M.D. Dong, "The core/shell composite nanowires produced by self-scrolling carbon nanotube onto copper nanowires", ACS Nano, Vol. 3, pp. 2235-2240, 2009.

[8] D. Xia, Q.Z. Xue, J. Xie, H.J. Chen, C. Lv, F. Besenbacher, M.D. Dong, "Fabrication of carbon nanoscrolls from monolayer graphene", Small, Vol. 6, pp. 2010-2019, 2010.

[9] B. Yurdumakan, N.R. Raravikar, P.M. Ajayan, A.Dhinojwala, "Synthetic gecko-foot hairs from multiwalled carbon nanotubes", Chem. Commun., Vol. 30, pp. 3799-3801, 2005.

[10] K. Autumn, Y.A. Liang, S.T. Hsieh, W. Zesch, W.P. Chan, T.W. 
Kenny, R. Fearing, R.J. Full, "Adhesive force of a single gecko-foot hair", Nature, Vol. 405, pp. 681-685, 2000.

[11] N. Yao, V. Lordi, "Carbon nanotube caps as springs: Molecular dynamics simulations", Phys. Rev. B, Vol. 58, pp. 12649-12651, 1998

[12] A. Grag, S.B. Sinnott, "Molecular dynamics of carbon nanotube proximal probe tip-surface contacts", Phys. Rev. B, Vol. 60, pp. 1378613791, 1999

[13] W.H. Duan, Q. Wang, K.M. Liew, X.Q. He, "Molecular mechanics modeling of carbon nanotube fracture", Carbon, Vol. 45, pp. 17691776, 2007. 